Advances in Computational Sciences and Technology

ISSN 0973-6107 Volume 10, Number 6 (2017) pp. 1861-1866

(C) Research India Publications

https://dx.doi.org/10.37622/ACST/10.6.2017.1861-1866

\title{
Economical Home Automation System using Arduino UNO
}

\author{
Naresh Kumar \\ Assistant Professor, MSIT, New Delhi, India. \\ Praveer Singh \\ B. Tech. Student, MSIT, New Delhi,
}

\begin{abstract}
Home automation or smart home industry have seen dramatic growth in past years and will continue to in future as per the market requirements and it benefits. In the proposed paper we have tried to develop a home automation system that is affordable than the available similar products in market. Smart home or home automation involves the controlling and automation of electrical appliances such as lights, fans, heating, air-conditioner and other home appliances such as refrigerator, dish washer etc. that uses Bluetooth or Wi-Fi. Home automation system consists switches and sensors connected to a central hub from which the systems is controlled with a user-interface that is interacted via a wall mounted terminal, mobile phone software or via web interface.
\end{abstract}

Keywords: Automation, Arduino UNO, Sketch, Android.

\section{INTRODUCTION}

In the present day, automation has become one of the eminently attractive areas that play an important role in day to day life [1]. Home automation have various applications such as: Lighting control system, Appliance control and integration, Security, Leak and smoke detection, Home automation for the elderly and disabled etc. 
In recent decade market of home automation systems have seen immense growth round the world. Home energy consumption tends to grow in proportion to increase of large-sized home appliances. Energy usage must be improved to reduce carbon dioxide emissions [8].

Over the last ten years, many organizations within IT, Communication, Real Estate, and Home Appliances industry have been looking to identify and capitalize on the promise of opportunities in information technology at home. The concept of home information varies in different industries. The final goals of home information system are to provide family with various services and applications in order to make family life enjoyable, comfortable, convenient, and secure [9].

The home network composed within your home is providing home automation service and home viewer service by being connected with wire or wireless network equipment including household electric appliances such as refrigerator, microwave oven, A/V equipment, electric lamp etc. based on power line communication and camera based on UPnP[7].

In the proposed architecture we have tried to try to develop a home automation system that can perform similar operations to the existing products at significantly low cost. The system uses Bluetooth technology which is in built feature of a smart phone and provides a better wireless communication medium. An android application is developed to control the appliances and transmit the data over network.

\section{RELATED WORK}

In [1] architecture for home control and monitoring system using Android based smart phone is proposed and implemented. This architecture utilizes a micro web server and Bluetooth communication as an interoperable application layer for communicating the remote user and the home devices is proposed.

In [2] design and features of a Smart Home Automation System have been shown. It is Bluetooth based, hence wireless and can be flexible. It has a special feature for smart speech sense, which would decode users' sentences into appropriate commands.

The purpose of the system in [3] is to use mobile phones' inbuilt facility for automation. The paper describes different hardware and software units. The complete software has been designed using Android using $\mathrm{C}$ language.

In [4] the smart home automation based system is designed which consists of mobile phone and GSM modem. In this system, design, incoming message is sent from the user phone to GSM model as a text message via cellular network.

In [6] gives a novel architecture for a home automation system which is proposed and implemented, using the relatively new communication technology ZigBee. The use of 
ZigBee communications technology helps lower the expense of the system and the intrusiveness of the respective system installation.

\section{PROBLEM FORMULATION}

The main problems formulated in $[1,2,3]$ are:

a) The system developed in [1] uses multiple modules and services in a single system making the system more complex and results in low performance. A use of server, Wi-Fi and Bluetooth in a single system also enhances the cost of the complete system.

b) In [2] the system uses smart speech sense which would decode users' sentence into appropriate commands. It requires strong authentication mechanism as a medium of security and a need for preventing the use of application by unauthorized user.

\section{PROPOSED WORK}

The Figure 1 shows the proposed architecture of Economical Home Automation System using Arduino. The modules are shown below:

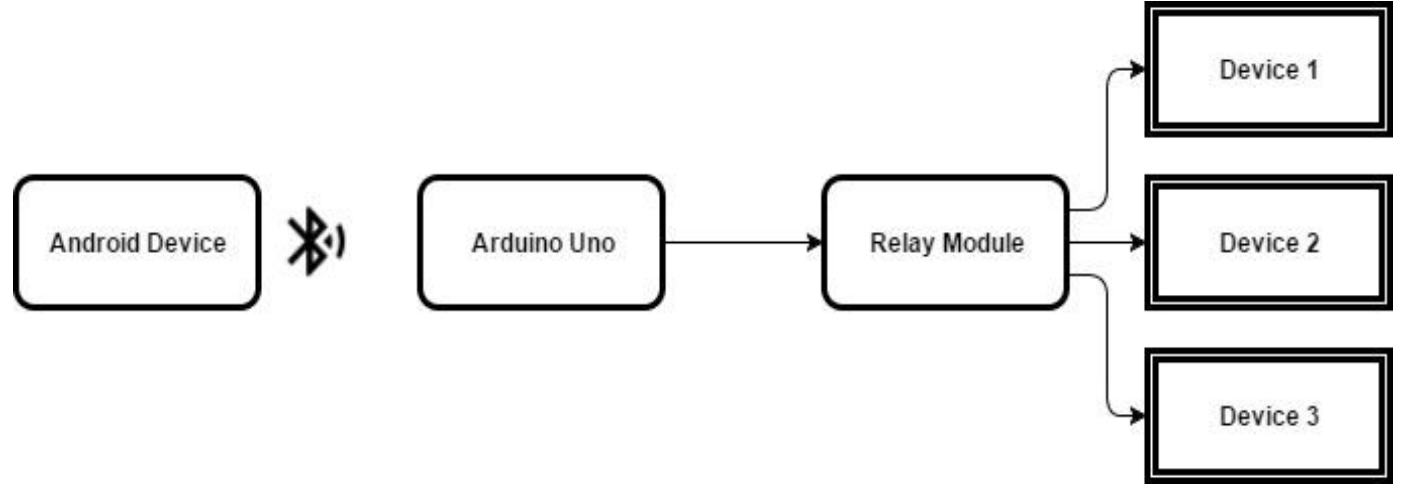

Figure 1: System Architecture

a) Arduino UNO: A sketch is a program written in a high level language to run the Arduino Uno. A sketch is written which makes compatibility among the microcontroller, Bluetooth adapter and the android application which controls the behavior of the device on the actions performed by user. The detailed algorithm is shown in Figure 2. 


\section{Algorithm: Arduino Sketch Algorithm.}

Input: Character 'received' from the android application.

Output: Status of pin.

1. Initialize INPUT OUTPUT pins and an array 'pins' for track of status pins in setup().

2. If Serial input is available start listening to serial data.

2.1 Read serial data and store it into a variable 'received'.

2.2 Switch(received)

2.2.1 Case 'A': Set PIN 2 HIGH.

2.2.2 Case 'a': Set PIN 2 LOW.

2.2.3 Case 'B': Set PIN 2 HIGH.

2.2.4 Case 'b': Set PIN 2 LOW.

2.2.5 Case ' $C$ ': Set PIN 2 HIGH.

2.2.6 Case 'c': Set PIN 2 LOW.

2.2.7 Case 'D': Set PIN 2 HIGH.

2.2.8 Case 'd': Set PIN 2 LOW.

2.2.9 Case 'E': Set PIN 2 HIGH.

2.2.10 Case 'e': Set PIN 2 LOW.

2.2.11 Case 'F': Set PIN 2 HIGH.

2.2.12 Case 'f': Set PIN 2 LOW.

2.2.13 Case ' $G$ ': Set PIN 2 HIGH.

2.2.14 Case 'g': Set PIN 2 LOW.

2.2.15 Case 'H': Set PIN 2 HIGH.

2.2.16 Case ' $h$ ': Set PIN 2 LOW.

2.2.17 Case 'I': Set PIN 2 HIGH.

2.2.18 Case 'i’: Set PIN 2 LOW.

2.2.19 Case 'J': Set PIN 2 HIGH.

2.2.20 Case 'j': Set PIN 2 LOW.

2.2.21 Case 'K': Set PIN 2 HIGH.

2.2.22 Case 'k': Set PIN 2 LOW.

2.2.23 Case 'L': Set PIN 2 HIGH.

2.2.24 Case '1': Set PIN 2 LOW.

2.2.25 Case 'T': Set all PINs HIGH.

2.2.26 Case 't': Set all PINs LOW.

2.2.27 Case 'S': Send status of all PINs.

2.3 Set received equals to null.

3. If power is on go to 2 .

4. Else End.

Figure 2: Algorithm: Arduino Sketch Algorithm. 
b) Android Application Development: An android application is developed to control the device remotely. The application contains the button corresponding to the devices present and a Bluetooth button is used to first make connection between the mobile phone and the microcontroller and device can be turned on or off by pressing the button icon in the android application.

c) Relay Module: A relay is an electrically operated switch operated by a low electric current that can control a device operating on much larger electric current. A relay module is a hardware device that consists of other electronic components to make a relay compatible for the devices to be operated as well as the Arduino UNO circuit board.

d) Bluetooth Connectivity: For connecting the devices to be automated, the Bluetooth technology is used using HC-06 Bluetooth module. The Bluetooth provides the simplest connection environment and does not require any server access and avoids many more complexities which are achieved if the Wi-Fi technology is used.

\section{DISCUSSION OF RESULTS}

The system provides automated home appliances and the device developed for this purpose is cost reduction that can be seen from the below parameters:

Table 1: Price of components used

\begin{tabular}{|l|c|}
\hline Components & Price(in Rupees) \\
\hline Bluetooth module & 350 \\
\hline Relay & 22 \\
\hline Arduino Uno & 400 \\
\hline Other components (Resistors, Transistors etc.) & 400 \\
\hline Total & $\mathbf{1 1 7 2}$ \\
\hline
\end{tabular}

In table 1, prices of each module and miscellaneous items such as resistors transistors, diodes, PCB etc. is shown. The price of complete unit comes around $\sim$ Rs. 1200 or $\$ 17$. In consumer market, home automation systems available are of much higher cost than the proposed system. Thus, proposed architecture can make home automation system much more affordable, so that more consumers can adopt it. 


\section{CONCLUSION}

The proposed project provides a Home Automation System that uses Bluetooth technology of a smart phone with the help of an android application. The project provides a user friendly automation system with low cost and good performance. The user gives command through the android application which is connected to Arduino Uno with the help of Bluetooth then the electrical appliances of a home can be regulated.

\section{REFERENCES}

[1] David N. et al., "Design of Home Automation", International Journal of Scientific \& Engineering Research, Volume 6, Issue 6, 2015, ISSN 2229-5518

[2] Pandya B. et al., "Android based Home Automation System using Bluetooth \& Voice Command" in International Research Journal of Engineering and Technology (IRJET) Volume: 03 Issue: 03, 2016, ISSN: 2395-0072

[3] Dmello A. et al., " Home Automation using Raspberry Pi 2, "International Journal of Current Engineering and Technology, Vol.6, No. 3, 2016, E-ISSN $2277-4106$.

[4] Teymourzadeh R. et al., "Smart GSM Based Home Automation System", IEEE Conference on Systems, Process \& Control (ICSPC2013)Kuala Lumpur, Malaysia, 2013

[5] QutabBaig M. et al. "A Comparative Analysis on Home Automation Techniques" , Second International Conference on Artificial Intelligence, Modelling and Simulation, 2014, DOI: 10.1109/AIMS.2014.11

[6] Gill K. et al.," A ZigBee-Based Home Automation System" in IEEE Transactions on Consumer Electronics, Vol. 55, No. 2, 2009, DOI: 10.1109/TCE.2009.5174403.

[7] Ok S. and H. Park, "Implementation of initial provisioning function for home gateway based on open service gateway initiative platform", in 8th International Conference on Advanced Communication Technology, pp. 15171520, 2006, ISBN 89-5519-129-4.

[8] Kushiro N. et al., "Integrated home gateway controller for home energy management system", IEEE International Conference on Consumer Electronics, pp.386-387, 2003,DOI: 10.1109/TCE.2003.1233787.

[9] Pu L., "An Improved Short Message Security Protocol for Home Network,", Proc. 2009 International Conference on Future Computer and Communication (FCC '09), Wuhan, pp.62-65, 2009,DOI: 10.1109/FCC.2009.12 